\title{
A study on subarcsecond scales of the ammonia and continuum emission toward the G16.59-0.05 high-mass star-forming region
}

\author{
L. Moscadelli ${ }^{1}$, R. Cesaroni ${ }^{1}$, Á. Sánchez-Monge ${ }^{1}$, C. Goddi ${ }^{2}$, R. S. Furuya ${ }^{3}$, A. Sanna ${ }^{4}$, and M. Pestalozzi ${ }^{5}$ \\ ${ }^{1}$ INAF - Osservatorio Astrofisico di Arcetri, Largo E. Fermi 5, 50125 Firenze, Italy \\ e-mail: mosca@arcetri.astro.it \\ 2 Joint Institute for VLBI in Europe, Postbus 2, 7990 AA Dwingeloo, The Netherlands \\ 3 The University of Tokushima Minami Jousanjima-machi 1-1, Tokushima, Tokushima 770-8502, Japan \\ ${ }^{4}$ Max-Planck-Institut für Radioastronomie, Auf dem Hügel 69, 53121 Bonn, Germany \\ 5 INAF - Istituto Fisica Spazio Interplanetario, via Fosso del Cavaliere 100, 00133 Roma, Italy
}

Received 24 May 2013 / Accepted 15 September 2013

\begin{abstract}
Aims. We wish to investigate the structure, velocity field, and stellar content of the G16.59-0.05 high-mass star-forming region, where previous studies have established the presence of two almost perpendicular (NE-SW and SE-NW), massive outflows, and a rotating disk traced by methanol maser emission.

Methods. We performed Very Large Array observations of the radio continuum and ammonia line emission, complemented by COMICS/Subaru and Hi-GAL/Herschel images in the mid- and far-infrared.

Results. Our centimeter continuum maps reveal a collimated radio jet that is oriented E-W and centered on the methanol maser disk, placed at the SE border of a compact molecular core. The spectral index of the jet is negative, indicating non-thermal emission over most of the jet, except the peak close to the maser disk, where thermal free-free emission is observed. We find that the ammonia emission presents a bipolar structure consistent (on a smaller scale) in direction and velocity with that of the NE-SW bipolar outflow detected in previous $\mathrm{CO}$ observations. After analyzing our previous $\mathrm{N}_{2} \mathrm{H}^{+}(1-0)$ observations again, we conclude that two scenarios are possible. In one case both the radio jet and the ammonia emission would trace the root of the large-scale CO bipolar outflow. The different orientation of the jet and the ammonia flow could be explained by precession and/or a non-isotropic density distribution around the star. In the other case, the $\mathrm{N}_{2} \mathrm{H}^{+}(1-0)$ and ammonia bipolarity is interpreted as two overlapping clumps moving with different velocities along the line of sight. The ammonia gas also seems to undergo rotation consistent with the maser disk. Our infrared images complemented by archival data allow us to derive a bolometric luminosity of $\sim 10^{4} L_{\odot}$ and to conclude that most of the luminosity is due to the young stellar object associated with the maser disk.

Conclusions. The new data suggest a scenario where the luminosity and the outflow activity of the whole region could be dominated by two massive young stellar objects: 1) a B-type star of $\sim 12 M_{\odot}$ at the center of the maser/ammonia disk; 2) a massive young stellar object (so far undetected), very likely in an earlier stage of evolution than the B-type star, which might be embedded inside the compact molecular core and power the massive, SE-NW outflow.
\end{abstract}

Key words. techniques: interferometric - ISM: jets and outflows - ISM: molecules - radio continuum: ISM - infrared: ISM

\section{Introduction}

The study of high-mass $\left(M>8 M_{\odot}\right)$ star formation still faces fundamental questions. Among the most important issues still to be clarified are 1) the role played by accretion disks to convey mass onto the (proto)star; 2) the properties of the (proto)stellar outflows (neutral vs ionized; wide-angle vs collimated); 3 ) the way an HII region develops and expands. From a theoretical point of view, models of massive star formation are complicated by the need to consider the effects of the intense stellar radiation of a massive young stellar object (MYSO), which, by heating, ionizing and exerting pressure on the circumstellar gas, strongly influences the process of mass accretion and ejection (e.g., Peters et al. 2010; Cunningham et al. 2011). Models predict that an HII region is quenched, remains trapped or expands hydrodynamically, depending on the balance between the stellar radiation and thermal pressure, on one hand, and the gravitational pull of the massive (proto)star and the ram pressure of the infalling material, on the other (Keto 2002, 2003).
From an observational point of view, the large (typically several $\mathrm{kpc}$ ) distances of massive stars and their origin in clusters make it difficult to disentangle the physical and kinematical properties of a single massive (proto)star from those of other cluster members. Prior to the advent of the Atacama Large Millimeter Array (ALMA), the angular resolution ( $\geq$ a few $0 . ' 1$ ) of millimeter interferometers (PdBI, SMA) was in most cases inadequate for resolving massive accretion disks (with modelpredicted sizes of a few hundred AU) and identify the protostellar outflow emitted from a "single" MYSO (hidden in the complex emission pattern resulting from the interacting outflows of the cluster). This can explain the failure to detect disks in O-type stars and the small number of bona-fide detections obtained for B-type stars (Cesaroni et al. 2007; Sánchez-Monge et al. 2013a). Use of the Very Large Array (VLA) has permitted the detection of weak $(\sim 1-10 \mathrm{mJy})$, relatively compact (size $\left.\leq 1^{\prime \prime}\right)$, thermal continuum sources nearby MYSOs, which has been recognized as an important tool for investigating accretion and ejection phenomena. The origin of such emission has been interpreted in terms of photo-ionized spherical (Panagia \& Felli 1975) and 
collimated (Reynolds 1986) stellar winds, shock-induced radiation (Ghavamian \& Hartigan 1998), and, more recently, trapped HII regions (Keto 2003). See also Rodríguez et al. (2012) and Sánchez-Monge et al. (2013b) and references therein, for a list of different mechanisms proposed to explain the observation of thermal centimeter continuum emission in star-forming regions.

With this in mind, in the past years we have focused on the high-mass star-forming region G16.59-0.05, also known as IRAS 18182-1433. This object has been investigated by various authors via thermal line interferometric (SMA, OVRO, PdBI) and maser VLBI observations (Beuther et al. 2006; Furuya et al. 2008; Sanna et al. 2010, hereafter B2006, F2008, S2010, respectively). Its distance $(3.6 \pm 0.3 \mathrm{kpc})$ has been recently determined with maser trigonometric parallax observations (Zhang, priv. comm.). Inside a molecular clump of $\sim 0.5 \mathrm{pc}$ and $\sim 1900 M_{\odot}$ (Beuther et al. 2002a; Hill et al. 2005), millimeter continuum and line observations reveal a number of young stellar objects (YSOs) and a complex flow pattern, dominated by two almost perpendicular (NE-SW and SE-NW), massive outflows (see Fig. 8 of B2006). 18.1 $\mu \mathrm{m}$ emission from the molecular clump is marginally detected by De Buizer et al. (2005) with the $3 \mathrm{~m}$ NASA Infrared Telescope Facility. The VLA C-array observations at $3.6,1.3$ and $0.7 \mathrm{~cm}$ of Zapata et al. (2006) identify three compact continuum sources, named "a", "b" and "c", within a distance of $\approx 10$ ". Sources "a" and "b" belong to the molecular clump and are separated by $\sim 2$ " along the SE-NW direction: source "a", the one to NW, is detected only at $0.7 \mathrm{~cm}$, whereas source "b", to SE, is visible both at 3.6 and $1.3 \mathrm{~cm}$. Interferometric measurements at $3 \mathrm{~mm}$ (F2008) and $1 \mathrm{~mm}$ (B2006) show that the continuum emission, engulfing both "a" and " $b$ " sources, peaks at the position of source "a", indicating that this source is the most embedded, and plausibly youngest object in the clump. The SMA detection of highdensity molecular tracers (B2006) with rotational temperatures as high as $130-150 \mathrm{~K}$ (inferred from the $\mathrm{CH}_{3} \mathrm{CN}$ lines by $\mathrm{F} 2008$ ) close to the position of " $b$ ", indicates that this centimeter continuum source is likely associated with a hot molecular core (HMC). For the sake of simplicity, hereafter we will refer to source "a" as mm-core and to source "b" as HMC.

Intense ( $\geq 10 \mathrm{Jy}$ ) Class II $6.7 \mathrm{GHz}$ and Class I $44.1 \mathrm{GHz}$ methanol and $22 \mathrm{GHz}$ water masers have been detected toward the G16.59-0.05 star-forming region. The European VLBI Network (EVN) observations of S2010 demonstrate that the $6.7 \mathrm{GHz} \mathrm{CH}_{3} \mathrm{OH}$ masers are associated with the $\mathrm{HMC}$ and trace an elongated structure of $\sim 2000 \mathrm{AU}$, with the 3D maser velocity pattern suggesting rotation (see Fig. 7 of S2010) about a mass $^{1}$ of $\sim 12 M_{\odot}$, assuming centrifugal equilibrium. Moscadelli et al. (2011) find that most of the $6.7 \mathrm{GHz}$ maser features present a regular internal $V_{\text {LSR }}$ gradient, which can be also interpreted in terms of Keplerian rotation around a star having a similar position and mass as derived from the maser 3D velocity distribution. Water masers, monitored with the Very Long Baseline Array (VLBA) by S2010, appear distributed close to the HMC, with measured proper motions tracing fast $\left(\approx 50 \mathrm{~km} \mathrm{~s}^{-1}\right)$ and poorly collimated expansion to the west (see Fig. 5b of S2010). A sketch of the main emission features in the G16.59-0.05 starforming region is presented in Fig. 8 of S2010, which illustrates

\footnotetext{
1 The mass quoted here differs from that computed by $\mathrm{S} 2010$ (35 $\left.M_{\odot}\right)$, since we have taken into account the new distance estimate of $d=$ $3.6 \mathrm{kpc}$ (instead of $4.4 \mathrm{kpc}$ ), and because of slightly different assumptions concerning the systemic velocity adopted and the maser spots used for the calculation (2-3 spots might not be tracing the disk). Our new estimate of $\sim 12 M_{\odot}$ uses a rotation velocity of $5.1 \mathrm{~km} \mathrm{~s}^{-1}$ (for a systemic velocity of $60 \mathrm{~km} \mathrm{~s}^{-1}$ ) at a radius of $0 !^{\prime} 11$.
}

the spatial distribution of the continuum sources, and thermal and maser line tracers.

This paper reports on new observations of G16.59-0.05 performed with the VLA and Subaru telescope at radio and infrared wavelengths. We have imaged both the continuum and ammonia line emission at subarcsecond resolution and complemented these data with Herschel continuum images from the Hi-GAL survey. Our purpose is to investigate the structure of the molecular and ionized gas close to the origin of the large scale molecular outflow(s) and establish the number and nature of the MYSOs associated with the maser disk and/or outflow(s). In the following, after illustrating the observations in Sect. 2, our findings will be presented and discussed in Sects. 3-5 and a scenario will be eventually proposed in Sect. 6 to explain in a consistent manner all the features of this intriguing high-mass star-forming region. Finally, conclusions are drawn in Sect. 7.

\section{Observations}

\subsection{Very Large Array}

\subsection{1. $\mathrm{NH}_{3}$ emission and $\mathrm{H}_{2} \mathrm{O}$ masers}

We observed the $\mathrm{NH}_{3}(1,1)$ (at rest frequency of $23694.5060 \mathrm{MHz}$ ) and $\mathrm{NH}_{3}(2,2)$ (at $23722.6336 \mathrm{MHz}$ ) main lines, and the water maser emission (at $22235.08 \mathrm{MHz}$ ) in G16.59-0.05 with the NRAO VLA ${ }^{2}$ in the B-Array configuration (project code 12A-054) in three different runs of two hours on July 5, July 30, and August 6 2012. We recorded dual polarization using two IFs (each comprising eight adjacent $4 \mathrm{MHz}$ bandwidths), one centered at a rest frequency $(23708.57 \mathrm{MHz}$ ) halfway between the $\mathrm{NH}_{3}(1,1)$ and $(2,2)$ main lines, and the other at the water maser sky frequency. Considering the VLA correlator capabilities in Summer 2012, this frequency setup was selected to observe both the $\mathrm{NH}_{3}$ and the water maser emission, to maximize the number of observable $\mathrm{NH}_{3}(1,1)$ and $(2,2)$ satellite lines and, at the same time, attain a high enough velocity resolution to resolve the $\mathrm{NH}_{3}$ lines. We could observe the $\mathrm{NH}_{3}(1,1)$ satellite lines with velocity separation (from the main line) of $\pm 7 \mathrm{~km} \mathrm{~s}^{-1}$ and $+19 \mathrm{~km} \mathrm{~s}^{-1}$, and the $\mathrm{NH}_{3}(2,2)$ satellite lines at $+16 \mathrm{~km} \mathrm{~s}^{-1}$ and $+26 \mathrm{~km} \mathrm{~s}^{-1}$. Correlating each $4 \mathrm{MHz}$ bandwidth with 128 channels, we achieved a velocity resolution of $0.39 \mathrm{~km} \mathrm{~s}^{-1}$.

The primary and phase calibrators were the VLA calibrators 3C286 and J1832-1035, respectively. The phase calibrator is separated from G16.59-0.05 on the sky by 4.8. Each 2-h run included a single 5 -min scan on the primary calibrator, eight 3-min scans on the phase calibrator, and a total of $1.1 \mathrm{~h}$ of on-source time. First, we applied the amplitude and phase corrections derived from the calibrators to the strongest (reference) water maser channel, and then, we self-calibrated the reference maser channel. Self-calibration was effective in improving the signal-to-noise ratio $(\mathrm{S} / \mathrm{N})$ of the image of the reference maser channel by a factor $\geq 10$. For each of the three observing runs, the calibration of the $\mathrm{NH}_{3}$ line, water maser and continuum emission of G16.59-0.05 consisted of two steps, applying first the calibrator amplitude and phase corrections, and then the selfcalibration solutions from the reference maser channel. The line data have been also corrected for the time variable Doppler shift (not accounted by the VLA correlator), which, during a single

2 The National Radio Astronomy Observatory is operated by Associated Universities, Inc., under cooperative agreement with the National Science Foundation. 
Table 1. Centimeter continuum fluxes for the VLA A and B-Array observations.

\begin{tabular}{lcccccc}
\hline \hline Wavelength & $\begin{array}{c}\theta_{\text {beam }} \\
\left({ }^{\prime \prime} \times \times^{\prime \prime}\right)\end{array}$ & $\begin{array}{c}\mathrm{PA}_{\text {beam }} \\
\left({ }^{\circ}\right)\end{array}$ & $\begin{array}{c}\mathrm{rms} \\
\left(\mathrm{mJy} \mathrm{beam}^{-1}\right)\end{array}$ & $\begin{array}{c}I_{\text {peak }} \\
\left(\mathrm{mJy} \mathrm{beam}^{-1}\right)\end{array}$ & $\begin{array}{c}S_{v}^{\text {thermal }} \\
(\mathrm{mJy})\end{array}$ & $\begin{array}{c}S_{v}^{\text {non-thermal }} \\
(\mathrm{mJy})\end{array}$ \\
\hline $6.0 \mathrm{~cm}-\mathrm{A}$ & $0.540 \times 0.330$ & -6 & 0.008 & 0.164 & $0.031 \pm 0.002$ & $0.434 \pm 0.015$ \\
$2.0 \mathrm{~cm}-\mathrm{A}$ & $0.241 \times 0.163$ & -14 & 0.009 & 0.117 & $0.099 \pm 0.005$ & $0.538 \pm 0.018$ \\
$1.3 \mathrm{~cm}-\mathrm{B}$ & $0.400 \times 0.400$ & +0 & 0.060 & 0.375 & $0.146 \pm 0.008$ & $\leq 0.612$ \\
$1.3 \mathrm{~cm}-\mathrm{A}$ & $0.131 \times 0.075$ & -173 & 0.023 & 0.265 & $0.324 \pm 0.032$ & $\leq 0.631$ \\
\hline
\end{tabular}

run, causes the line emission to drift by at most the velocity resolution channel.

Before imaging, the calibrated data of the three runs have been concatenated. We produced naturally-weighted and $u v$-tapered (Gaussian tapering with full width at half maximum (FWHM) of $400 \mathrm{k} \lambda$ ) images of the $\mathrm{NH}_{3}$ main and satellite lines, water maser and continuum emission. The continuum data was obtained by averaging the 13 (out of the 16) $4 \mathrm{MHz}$ bandwidths of the two IFs not containing $\mathrm{NH}_{3}(1,1)$ and $(2,2)$, nor the water maser lines. The naturally-weighted beam has a FWHM size of $0.48 \times 0.31$, at $\mathrm{PA}=7^{\circ}$, with small difference between the $\mathrm{NH}_{3}$ line and continuum maps. The continuum emission map, restored using a round beam with FWHM size of 0. '4 has a rms noise level of $0.06 \mathrm{mJy}_{\text {beam }}{ }^{-1}$. To increase the $\mathrm{S} / \mathrm{N}$ on the $\mathrm{NH}_{3}$ lines, before imaging, the data have been smoothed in velocity, degrading the velocity resolution to $0.8 \mathrm{~km} \mathrm{~s}^{-1}$. The typical rms noise on the channel maps of the $\mathrm{NH}_{3}$ main and satellite lines is of $1.3-1.5 \mathrm{mJy}^{\text {beam }}{ }^{-1}$.

\subsubsection{Continuum}

We used the VLA A-Array (code: 12B-044) to observe the continuum emission of G16.59-0.05 at $C$-, $K \mathrm{u}$ - and $K$-band (centered at $6.2,13.1$ and $21.7 \mathrm{GHz}$, respectively) in four different runs on October 2, October 21 and December 30 2012, and January 17 2013. G16.59-0.05 was observed for $15 \mathrm{~min}$ at $C$ and $\mathrm{Ku}$-band, and for $30 \mathrm{~min}$ at $K$-band. At all bands, the primary and phase calibrators were the VLA calibrators 3C286 and J1832-1035, respectively. We employed the new capabilities of the WIDAR correlator which permits us to record dual polarization using four IFs each comprising eight adjacent $128 \mathrm{MHz}$ subbands, achieving a total bandwidth per polarization of $2 \mathrm{GHz}$. At $6 \mathrm{GHz}$ and $22 \mathrm{GHz}$, we centered one IF at the frequency of the methanol and water maser, respectively. Correlating each $128 \mathrm{MHz}$ subband with 128 channels, we achieved a velocity resolution of $45 \mathrm{~km} \mathrm{~s}^{-1}$ and $13 \mathrm{~km} \mathrm{~s}^{-1}$ at $6 \mathrm{GHz}$ and $22 \mathrm{GHz}$, respectively. Since the methanol and the water maser signals in G16.59-0.05 are strong $\left(\approx 20 \mathrm{Jy} \mathrm{beam}^{-1}\right)$ and relatively wide $\left(\approx 10 \mathrm{~km} \mathrm{~s}^{-1}\right)$, we could detect them with high $(\geq 100) \mathrm{S} / \mathrm{N}$ even with these coarse velocity resolutions.

At $\mathrm{Ku}$-band, the amplitude and phase corrections of the G16.59-0.05 visibilities have been derived working only with the calibrators. At $C$ - and $K$-band, we first applied the calibrator corrections to the maser channel and self-calibrated the maser emission (improving the $\mathrm{S} / \mathrm{N}$ of the maser image by a large factor $(\geq 10)$ ), and then, before mapping, applied the self-calibration solutions from the maser to the continuum data. In each band, the rms noise level of the continuum image is close to the expected thermal noise: $8 / 9 \mu \mathrm{Jy}^{b^{2}}$ bem $^{-1}$ at $6 / 14 \mathrm{GHz}$ and $23 \mu \mathrm{Jy} \mathrm{beam}^{-1}$ at $22 \mathrm{GHz}$. The FWHM size of the naturally-weighted beams at the different observing wavelenghts are listed in Table 1.

\subsection{Subaru}

Using the mid-infrared imaging spectrometer (COMICS; Kataza et al. 2000) at the Cassegrain focus of the $8.2 \mathrm{~m}$ Subaru Telescope, we carried out imaging observations of the $24.5 \mu \mathrm{m}$ emission toward G16.59-0.05 on 2008 July 15. For this purpose, we configured the $Q 24.5$ filter, and employed choppingand-nodding mode for subtracting the sky-background emission. The camera provides a field of view of $\sim 42^{\prime \prime} \times 32^{\prime \prime}$ with a pixel size of $0{ }^{\prime} 13$. Flux calibration was performed toward four standard sources listed in Cohen et al. (1999): HD 146051, HD 186791, HD 198542, and HD 198542. We estimate the overall uncertainty in the flux calibrations to be less than $10 \%$.

To perform the astrometric calibration of the image, we have compared the Subaru image with the MIPSGAL image at $24 \mu \mathrm{m}$ (Carey et al. 2009), after smoothing the former to the same angular resolution $\left(6^{\prime \prime}\right)$ as the latter. We have then aligned the two images by visually overlaying the non-saturated part of the MIPSGAL image on the corresponding part of the Subaru image. The original Subaru image was corrected by about $3^{\prime \prime}$. This process, albeit not very accurate, should result in an absolute positional uncertainty $\lesssim 1^{\prime \prime}$, comparable to the astrometric accuracy of MIPSGAL.

\section{Continuum emission: a bipolar jet}

From previous studies we know that radio continuum emission has been detected in several locations in the G16.59-0.05 region, but in the present study we will focus only on the emission associated with the HMC. Figure 1 presents a comparison among the continuum maps obtained by us at three different wavelengths and with different resolutions.

For the sake of comparison, we also show the $\mathrm{H}_{2} \mathrm{O}$ and $\mathrm{CH}_{3} \mathrm{OH}$ maser spots. The striking result is that, while the $1.3 \mathrm{~cm}$ emission appears compact and almost coincident with the $\mathrm{CH}_{3} \mathrm{OH}$ masers, consistent with Zapata et al. (2006) and S2010, at longer wavelengths $(2$ and $6 \mathrm{~cm}$ ) the emission appears elongated $\mathrm{E}-\mathrm{W}$ on both sides of the $\mathrm{CH}_{3} \mathrm{OH}$ maser spots, extending over $\sim 4^{\prime \prime}(0.07 \mathrm{pc})$. This morphology is strongly suggestive of a bipolar jet, whose powering source could lie close to the location of the $\mathrm{CH}_{3} \mathrm{OH}$ masers, near the geometrical center of the jet. In Table 1, we report the peak and integrated fluxes, derived from naturally-weighted maps, for the VLA A and B array observations. The thermal and non-thermal fluxes refer to the compact and extended continuum components, respectively, and are obtained by integrating the continuum emission inside and outside, respectively, the area where the $1.3 \mathrm{~cm}$ emission is $>5 \sigma$ (delimited by the white contour of Fig. 2c). The outer limit of the area where the non-thermal component has been computed, is determined by the $5 \sigma$ level of the $6 \mathrm{~cm}$ emission (see Fig. 2b).

Besides the morphology, a typical signature of thermal jets is their spectral index, ranging from -0.2 to +1.5 (see Anglada 1996) in the radio regime. To calculate the spectral index all over 


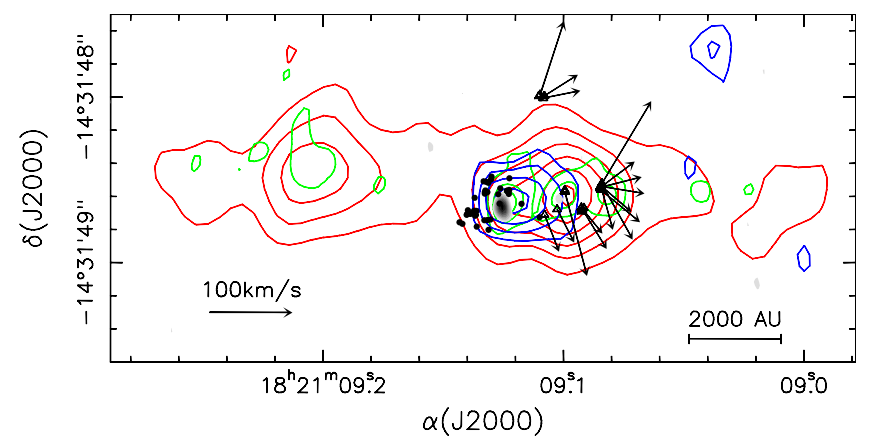

Fig. 1. Overlay of the radio continuum emission toward the HMC in G16.59-0.05 at $6 \mathrm{~cm}$ (red contours), $2 \mathrm{~cm}$ (green), $1.3 \mathrm{~cm}$ B-array (blue), and $1.3 \mathrm{~cm} \mathrm{A-array} \mathrm{(gray} \mathrm{scale).} \mathrm{The} \mathrm{contour} \mathrm{levels} \mathrm{range,}$ respectively, from 0.0264 to 0.1584 in steps of $0.0264 \mathrm{mJy}^{\text {beam }}{ }^{-1}$, from 0.03 to 0.09 in steps of $0.03 \mathrm{mJy} \mathrm{beam}^{-1}$, from 0.174 to 0.348 in steps of $0.058 \mathrm{mJy} \mathrm{beam}^{-1}$, and from 0.075 to 0.25 in steps of $0.025 \mathrm{mJy}_{\text {beam }}{ }^{-1}$. The solid circles and empty triangles represent, respectively, the $\mathrm{CH}_{3} \mathrm{OH}$ and $\mathrm{H}_{2} \mathrm{O}$ masers from $\mathrm{S} 2010$, while the arrows are the proper motions of the $\mathrm{H}_{2} \mathrm{O}$ masers. The scale for the proper motion amplitude is given on the bottom left of the panel. The size of the VLA beams for the different array configurations and wavelengths are reported in Table 1.

the continuum emission in G16.59-0.05, we have created maps of the 6,2 , and $1.3 \mathrm{~cm}$ continuum emission using the same $u v$ range for all bands, as well as the same clean beam $\left(0 .{ }^{\prime} 4 \times 0.0^{\prime} 4\right)$. In this way it is possible to compare the emission at different wavelengths minimizing the effects of different samplings of the uv plane and thus obtaining a more reliable estimate of the spectral index. The new maps are shown in the top panel of Fig. 2, while in the other two panels we show the maps of the spectral index $\alpha$ (assuming $S_{v} \propto v^{\alpha}$ ) between 6 and $2 \mathrm{~cm}$ (Fig. 2b) and between 2 and $1.3 \mathrm{~cm}$ (Fig. 2c). For these two estimates the uncertainty is, respectively, \pm 0.2 and \pm 0.5 . Note that $\alpha$ has been calculated only where the $6 \mathrm{~cm}$ (for Fig. 2b) and $2 \mathrm{~cm}$ (for Fig. 2c) fluxes are $>5 \sigma$. Moreover, the white contour encompasses the region where the $2 \mathrm{~cm}$ (for Fig. 2b) and $1.3 \mathrm{~cm}$ (for Fig. 2c) fluxes are $>5 \sigma$. This means that the values of the spectral indices lying outside the white contour are upper limits.

The striking result is that the spectral index between 6 and $2 \mathrm{~cm}$ is $<-0.5$ over a significant fraction of the region where $6 \mathrm{~cm}$ emission is detected. Such negative values are inconsistent with free-free emission and strongly suggestive of non-thermal emission. Indeed, this is not the first example of a radio jet with negative spectral index, associated with a massive (proto)star. Synchrotron emission has been proposed to explain the radio emission in $\mathrm{W} 3\left(\mathrm{H}_{2} \mathrm{O}\right)$ (Reid et al. 1995) and, more recently, in HH 80-81 (Carrasco-González et al. 2010), indicating that magnetic fields may play an important role in shaping such jets and, more in general, the accretion/ejection process in massive (proto)stars. In the case of G16.59-0.05 we find that: (i) the value of $\alpha$ in Fig. 2b is negative all over the region, but becomes $>-0.1$ (i.e., consistent with free-free emission) close to the $\mathrm{CH}_{3} \mathrm{OH}$ masers; (ii) the spectral index is $\sim 2$ between 2 and $1.3 \mathrm{~cm}$ (see Fig. 2c), as expected for optically thick free-free emission. However, at larger distance from the $\mathrm{CH}_{3} \mathrm{OH}$ masers (outside the white contour of Fig. 2c), the value of spectral index between 2 and $1.3 \mathrm{~cm}$ is only an upper limit and could be as low as measured between 6 and $2 \mathrm{~cm}$. Closer to the $\mathrm{CH}_{3} \mathrm{OH}$ masers, given the low brightness temperature measured at $1.3 \mathrm{~cm}$ $(69 \pm 6 \mathrm{~K}$ with the A-array), it is unlikely that the emission is optically thick and we believe that values of $\alpha$ as large as $\sim 2$ may

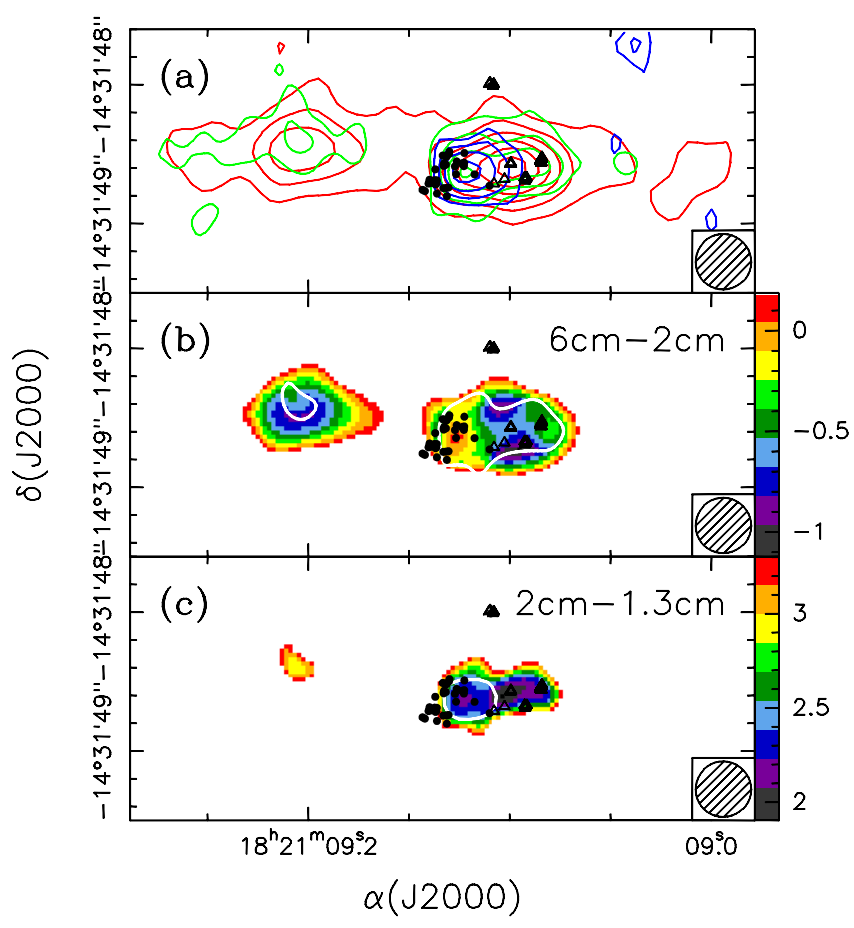

Fig. 2. a) Overlay of the VLA maps of the radio continuum emission at 6,2 , and $1.3 \mathrm{~cm}$ obtained using the common uv range and the same clean beam (shown in the bottom right) at all wavelengths. Contours and symbols have the same meaning as in Fig. 1. The contour levels range from 0.0264 to 0.1584 in steps of $0.0264 \mathrm{mJy} \mathrm{beam}^{-1}$ at $6 \mathrm{~cm}$, from 0.03 to 0.12 in steps of $0.03 \mathrm{mJy}_{\text {beam }}^{-1}$ at $2 \mathrm{~cm}$, and from 0.174 to 0.348 in steps of $0.058 \mathrm{mJy} \mathrm{beam}^{-1}$ at $1.3 \mathrm{~cm}$. b) Map of the spectral index between 6 and $2 \mathrm{~cm}$, computed where the $6 \mathrm{~cm}$ emission is $>5 \sigma$. The white contour encompasses the region where also the $2 \mathrm{~cm}$ emission is $>5 \sigma$. The values of the spectral index lying outside the white contour are upper limits. c) Same as panel b), for the spectral index between 2 and $1.3 \mathrm{~cm}$.

be partly due to the uncertainty caused by the proximity between the two wavelengths used for the calculation.

Nonetheless, it is clear that the difference between $\alpha(6-2 \mathrm{~cm})$ and $\alpha(2-1.3 \mathrm{~cm})$ is real. A possible explanation is that the radio emission is made of two contributions, with synchrotron dominating at long wavelengths and at larger separations from the powering MYSO, and bremsstrahlung preponderant close to the MYSO. Note infact that, near the $\mathrm{CH}_{3} \mathrm{OH}$ masers, $\alpha$ is consistent with free-free at all frequencies. Interestingly, this is also where the most compact $1.3 \mathrm{~cm}$ emission is detected with the highest angular resolution (see gray scale in Fig. 1), which suggests that this compact emission could be tracing the dense ionized gas in the neighborhoods of the star powering the jet.

What is the origin of the free-free emission? This could certainly arise from material ionized by shocks along the jet. However, one cannot exclude that ionization is due to the stellar photons. In this case, assuming optically thin emission, from the $1.3 \mathrm{~cm}$ continum flux measured with the B-array (0.69 mJy) one obtains a stellar Lyman continuum of $\sim 10^{45} \mathrm{~s}^{-1}$, corresponding to a zero-age main-sequence (ZAMS) luminosity of $\sim 4000 L_{\odot}$ and a stellar mass of $\sim 10 M_{\odot}$ (see Davies et al. 2011). According to Beuther et al. (2002b, see their Fig. 5), this implies a mass loss rate for the associated outflow of a few times $10^{-3} M_{\odot} \mathrm{yr}^{-1}$. It is questionable whether this value can be compared to those of the two $\mathrm{CO}$ outflows imaged on a larger scale by B2006, F2008, and López-Sepulcre et al. (2009), because the direction of the radio jet differs by $\sim 45^{\circ}$ from that of 


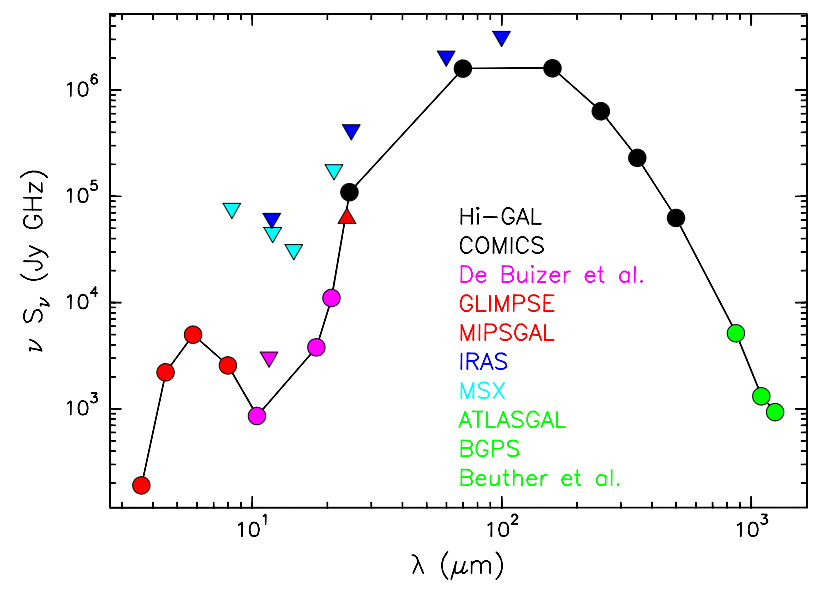

Fig. 3. Spectral energy distribution of G16.59-0.05 obtained from our IR measurements and archival data (see text). Triangles denote upper and lower limits. The luminosity estimate $\left(\sim 10^{4} L_{\odot}\right)$ has been obtained by integrating the SED under the solid line.

either outflow. It is hence possible that three massive objects are powering three distinct jets/outflows in the G16.59-0.05 region. We will come back to this issue in the following.

While the structure of this star-forming region is clearly very complex and will be further discussed in the next sections, we may conclude that our new radio continuum images strongly support the interpretation of the $\mathrm{CH}_{3} \mathrm{OH}$ masers as tracing a rotating disk (see S2010), because the radio jet appears to originate from a MYSO located close to the center of the maser spots and almost perpendicular to the elongated maser cluster.

\section{Bolometric luminosity and its origin}

Given the presence of multiple objects in G16.59-0.05, it is important to establish which of these are the main contributors to the luminosity of this star-forming region. We have reconstructed the spectral energy distribution (SED) using the following archival data: Galactic Legacy Infrared MidPlane Survey Extraordinaire (GLIMPSE; Benjamin et al. 2003), MIPSGAL/Spitzer $24 \mu \mathrm{m}$ (Carey et al. 2009), MSX (Egan et al. 2003), IRAS (Neugebauer et al. 1984), the APEX Telescope Large Area Survey of the Galaxy (ATLASGAL ${ }^{3}$; Schuller et al. 2009; Contreras et al. 2013), Bolocam Galactic Plane Survey (BGPS; Drosback et al. 2008), data from the literature (Beuther et al. 2002a; De Buizer et al. 2005), and recent PACS and SPIRE continuum images obtained in the context of the Herschel infrared Galactic Plane Survey (Hi-GAL; Molinari et al. 2010a,b).

The flux density estimates of IRAS, MSX, GLIMPSE, BGPS, and ATLASGAL were taken from the corresponding source catalogues, while those of Hi-GAL and MIPSGAL were computed from the images with aperture photometry, by integrating the emission inside suitable polygons encompassing our source and subtracting the background. Note that the MSX and IRAS flux densities are considered upper limits, because the HPBWs encompass a region significantly larger than that of interest for us. The MIPSGAL flux is a lower limit, because the image is partly saturated. We stress that such a limit is consistent with our Subaru measurement.

\footnotetext{
3 The ATLASGAL project is a collaboration between the MaxPlanck-Gesellschaft, the European Southern Observatory (ESO) and the Universidad de Chile.
}

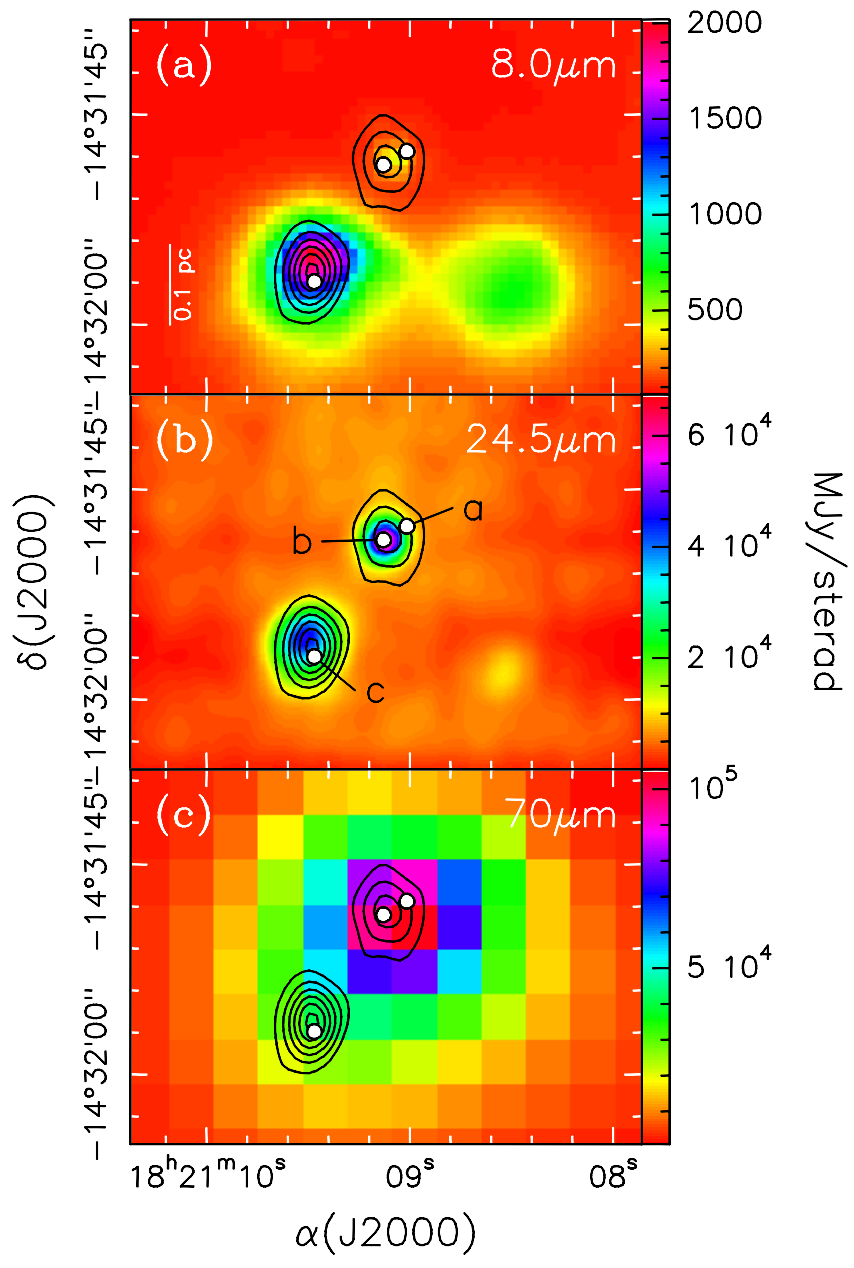

Fig. 4. a) Map of the continuum emission at $3.6 \mathrm{~cm}$ (black contours; from S2010) overlaid on an $8.0 \mu \mathrm{m}$ image (resolution $2^{\prime \prime}$ ) of the G16.59-0.05 region from the GLIMPSE/Spitzer survey (Benjamin et al. 2003). b) Same as panel a), for our Subaru $24.5 \mu \mathrm{m}$ image smoothed to the same angular resolution as the $8.0 \mu \mathrm{m} \mathrm{im}$ age (2"). c) Same as panel a), for the Hi-GAL/Herschel (Molinari et al. 2010b) $70 \mu \mathrm{m}$ image (resolution $\sim 10^{\prime \prime}$ ). The white dots indicate the positions of the centimeter sources "a" (alias mm-core), "b" (alias HMC) and "c" detected by Zapata et al. (2006).

To estimate the bolometric luminosity, the resulting SED (shown in Fig. 3) has been integrated by interpolating linearly between the known fluxes (solid line in the figure). We obtain $\sim 10^{4} L_{\odot}$, corresponding to a single star of $\sim 13 M_{\odot}$ (see Davies et al. 2011).

In the case of G16.59-0.05, the single-star assumption is inadequate to describe the situation, as three prominent IR sources are clearly detected over less than $\sim 20^{\prime \prime}$ (i.e., $\sim 0.3 \mathrm{pc}$ ). This is demonstrated by the $8 \mu \mathrm{m}$ GLIMPSE/Spitzer image, as well as our $24.5 \mu \mathrm{m}$ Subaru image, shown in Figs. $4 \mathrm{a}$ and $4 \mathrm{~b}$, respectively. The problem is that the angular resolution at $\lambda>70 \mu \mathrm{m}-$ i.e., close to the peak of the SED (see Fig. 3) - is not sufficient to resolve the three sources and find out which is responsible for most of the bolometric luminosity. To investigate this issue, in Fig. 5 we present a composite color image of the same region displayed in Fig. 4, where the blue, green, and red components are associated, respectively, with the $3.6,8$, and $24.5 \mu \mathrm{m}$ images (the first two from GLIMPSE/Spitzer). There is little doubt that the reddest and hence most embedded object is the northern source, namely the one coinciding with the HMC. Visual comparison of the three panels in Fig. 4 leads to the same conclusion, 


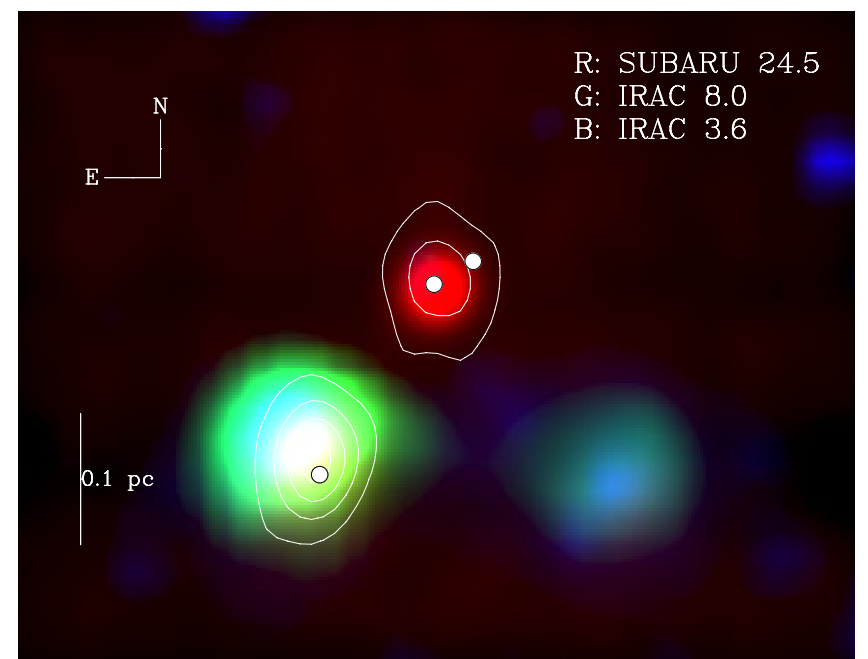

Fig. 5. Composite color image of the same field shown in Fig. 4, where blue is associated with the GLIMPSE/Spitzer $3.6 \mu \mathrm{m}$ image, green with the GLIMPSE/Spitzer $8.0 \mu \mathrm{m}$ image, and red with our $24.5 \mu \mathrm{m}$ Subaru image. The reddest and hence most extincted source is the one to the north, coinciding with the HMC. The VLA $3.6 \mathrm{~cm}$ continuum (from S2010) is shown with white contours. The white dots have the same meaning as in Fig. 4.

as the northern source becomes progressively more prominent with increasing wavelength, until it dominates the whole IR emission at $70 \mu \mathrm{m}$.

We conclude that in all likelihood the HMC is the source responsible for most of the $\sim 10^{4} L_{\odot}$ measured over the G16.59-0.05 region. However, one should keep in mind that, although the HMC is contributing to most of the IR emission (and hence of the bolometric luminosity), younger MYSOs might be embedded in the cold, dense surroundings of the HMC, possibly contributing significantly to the outflow activity in the region. We will come back to this point later.

\section{Ammonia emission}

Our ammonia observations provide us with information that is helpful to shed light on the structure and velocity field in this region. Such information is summarized by Fig. 6, which shows three overlays between the $\mathrm{NH}_{3}$ line emission and other relevant tracers.

In particular, in Fig. 6 a the integrated $\mathrm{NH}_{3}(1,1)$ line map is compared to the $24.5 \mu \mathrm{m}$ image obtained with Subaru, which confirms that the IR emission is tightly associated with the $\mathrm{HMC}$, within the positional uncertainty. It is also interesting to compare the $6 \mathrm{~cm}$ emission tracing the radio jet with the ammonia emission, as done in Fig. 6b. The strongest $6 \mathrm{~cm}$ continuum peak, which likely traces shocked gas of the radiojet (see Fig. 1 and Sect. 3), appears to coincide with the $\mathrm{NH}_{3}$ peak, while both are offset by $\sim 0^{\prime} .5(\sim 1800 \mathrm{AU})$ to the west of the $1.3 \mathrm{~cm}$ continuum peak and $\mathrm{CH}_{3} \mathrm{OH}$ masers - which likely pinpoint the position of the star powering the radio jet (see Fig. 1). This fact strongly suggests that the jet is impinging against dense molecular gas to the west, where the brightest radio emission is seen. This hypothesis is supported by the distribution and proper motions of the $\mathrm{H}_{2} \mathrm{O}$ masers (see Fig. 1), which are known to be associated with shocks. The proposed scenario could also explain why the jet appears slightly more extended to the east, as in this direction the gas would be much less dense.

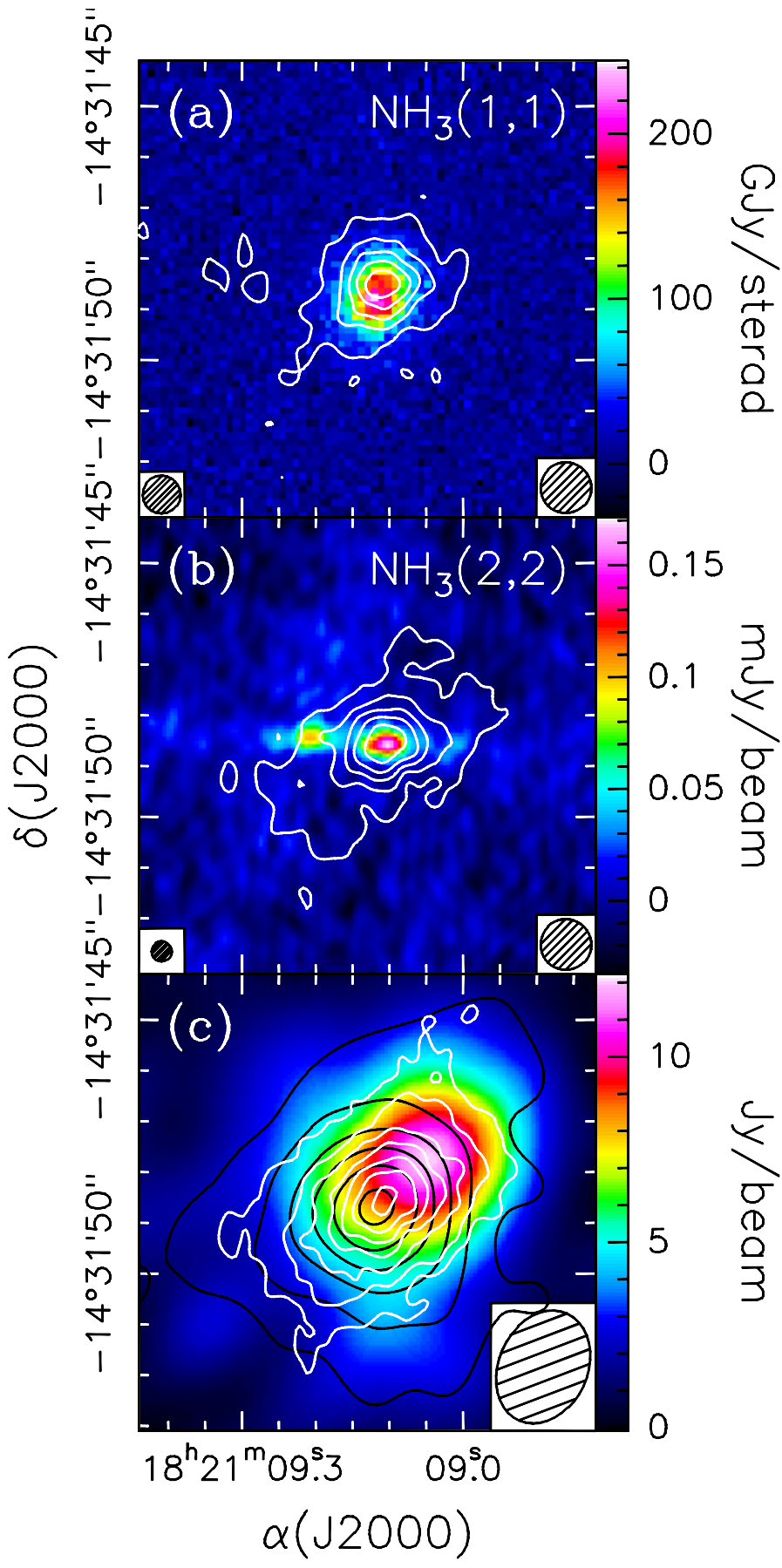

Fig. 6. a) Map of the $\mathrm{NH}_{3}(1,1)$ emission averaged over the main line (white contours) overlaid on an image of the $24.5 \mu \mathrm{m}$ continuum emission obtained with Subaru. The circles in the bottom left and right indicate, respectively, the angular resolution of the IR and radio data. b) Same as panel a), for the $\mathrm{NH}_{3}(2,2)$ line (contours) and the $6 \mathrm{~cm}$ radio continuum map (background image). c) Overlay between the $\mathrm{NH}_{3}(2,2)$ (white contours), $\mathrm{CH}_{3} \mathrm{CN}(5-4)$ (black contours), and $3.3 \mathrm{~mm}$ continuum (image) maps. The ammonia map has been smoothed to the same angular resolution (ellipse in the bottom right) as the methyl cyanide data (from F2008).

Figure $6 \mathrm{c}$ sheds further light on the structure of the region. This presents an overlay between our map of the $\mathrm{NH}_{3}(2,2)$ inversion line and the maps of the $\mathrm{CH}_{3} \mathrm{CN}(5-4)$ line and $3.3 \mathrm{~mm}$ continuum emission from F2008. Clearly, ammonia and methyl cyanide trace the same region (the HMC), as expected, while both are significantly offset (by $\sim 1$ ". 3 or $\sim 0.022 \mathrm{pc}$ ) from the 


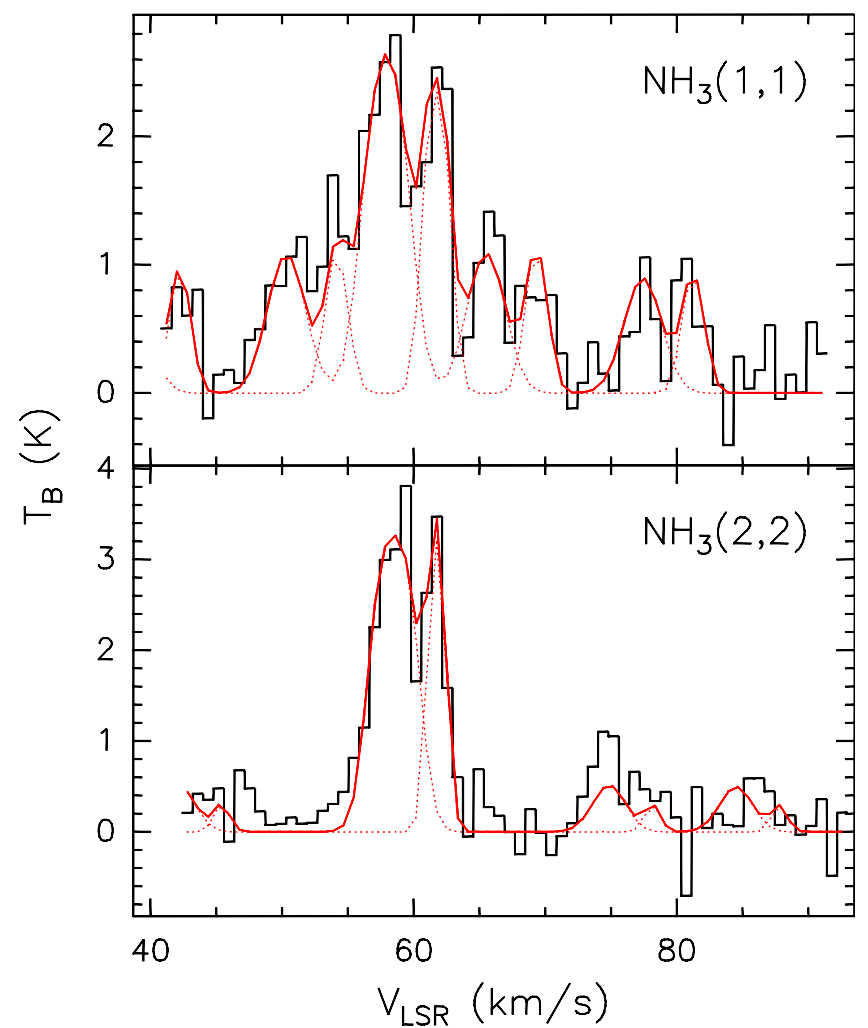

Fig. 7. Spectra of the $\mathrm{NH}_{3}(1,1)$ (top) and $(2,2)$ (bottom) inversion transitions obtained by averaging the emission over the HMC. The red solid curves are multiple Gaussian fits taking into account the hyperfine structure of the lines and two distinct velocity components (at about $58.2 \mathrm{~km} \mathrm{~s}^{-1}$ and $61.8 \mathrm{~km} \mathrm{~s}^{-1}$ ). The red dotted curves indicate the contribution of each velocity component to the total fit.

peak of the mm continuum, i.e., from the mm-core, as pointed out by B2006 and F2008.

All these features can be explained by a scenario where a massive star has formed close to the border of a dense molecular core (the mm-core) and has heated the surrounding gas, thus creating a HMC. This explains the offset between the HMC and the $\mathrm{mm}$-core. The latter must be quite massive, because it appears to dominate the mm continuum emission despite its low temperature ( $\sim 38 \mathrm{~K}$; see Williams et al. 2005) with respect to the HMC ( $\gtrsim 130 \mathrm{~K}$; see F2008). Using the same dust properties and continuum flux density (37 mJy) as in F2008, one can estimate a mass of $\sim 200 M_{\odot}$ for the mm-core. Such a large value suggests that other MYSOs, possibly in a much earlier evolutionary phase than the star in the HMC, might be forming inside the mm-core.

\subsection{Morphology and kinematics of the $\mathrm{NH}_{3}$ emission}

In Fig. 7, we show the average spectra of the $\mathrm{NH}_{3}(1,1)$ and $(2,2)$ inversion transitions over the HMC. The most intriguing feature is the presence of a double peak, clearly detected in both transitions. The fact that such a double peak is seen also in the optically thin satellites proves that this is not the effect of selfabsorption, but is due to two distinct velocity components. The red profiles in Fig. 7 are fits to the $\mathrm{NH}_{3}$ lines taking into account the hyperfine structure of the transitions, and clearly confirm that both the main line and satellite emission can be fit with two velocity components.

It is important to investigate the spatial distribution of these two components, as done in Fig. 8, where we compare the main

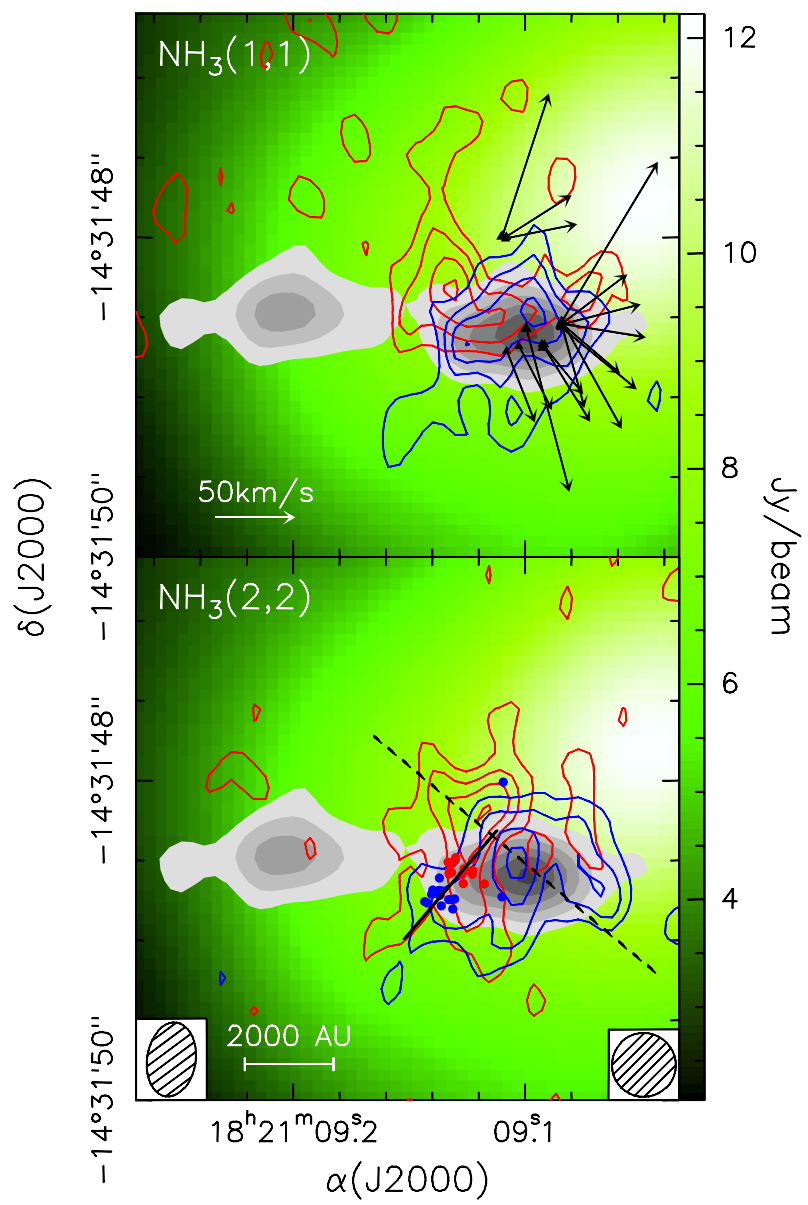

Fig. 8. Maps of the two velocity components (see Fig. 7) identified in the $\mathrm{NH}_{3}(1,1)$ (top) and $(2,2)$ (bottom) lines. Blue and red contours are the maps obtained by averaging the main line emission, respectively, from 55.8 to $60.6 \mathrm{~km} \mathrm{~s}^{-1}$ and from 60.6 to $63.0 \mathrm{~km} \mathrm{~s}^{-1}$. The gray scale is the same map of the $6 \mathrm{~cm}$ continuum emission as in Fig. 2a, while the backgroud image (green tones) is the map of the $3.3 \mathrm{~mm}$ continuum emission obtained by F2008. The red and blue dots give the position of the red- and blue-shifted, respectively, methanol masers from S2010. The black triangles and arrows are the water maser spots and corresponding proper motions from S2010. The amplitude scale for the proper motions is given on the bottom left of the upper panel. The continuous and dashed line in the lower panel indicate the axes of projection to produce the position-velocity plots of Figs. 10 and 11, respectively. The ellipses in the bottom left and right of the lower panel indicate, respectively, the $H P B W$ of the $6 \mathrm{~cm}$ continuum and ammonia line maps.

line $\mathrm{NH}_{3}(1,1)$ and $(2,2)$ emission integrated under the red and blue components, with the radio jet and the core traced, respectively, by the centimeter and millimeter continuum emission. For the sake of comparison, also the $\mathrm{CH}_{3} \mathrm{OH}$ and $\mathrm{H}_{2} \mathrm{O}$ masers are shown. We have used different colors for the blue- and the redshifted $\mathrm{CH}_{3} \mathrm{OH}$ spots, to outline the rotation of the disk about a $\mathrm{NE}-\mathrm{SW}$ axis. From this figure one sees that ammonia is tracing a compact bipolar structure roughly perpendicular to the $\mathrm{CH}_{3} \mathrm{OH}$ disk and slightly offset (by $\sim 0$.'4) to the NW with respect to it. Such a small offset (less than the $\mathrm{NH}_{3}$ map angular resolution) might be justified by the fact that the gas density is higher to the NW of the disk than to the SE.

The orientation and velocity of the ammonia structure being similar to the (larger scale) NE-SW bipolar outflow detected by B2006 and López-Sepulcre et al. (2009) suggests that both could be manifestations of the same ejection phenomenon. Indeed, this possibility cannot be excluded, as ammonia has been observed in 
association with bipolar jets in other massive sources (see, e.g., Zhang et al. 1999). In this scenario, the ammonia gas would be preferentially entrained by the flow on the NW side - i.e., that facing the core - where the density is higher. This would explain why the $\mathrm{NH}_{3}$ emission is slightly skewed to the NW.

\subsubsection{The outflow scenario}

To verify the outflow hypothesis, one may estimate the relevant parameters from the ammonia emission and compare them to those obtained for the larger-scale NE-SW bipolar outflow. We have computed the mass loss rate, momentum rate, and mechanical luminosity by integrating the blue- and red-shifted emission in the $\mathrm{NH}_{3}(1,1)$ main line over the respective lobes, and dividing by the dynamical time scale. In this calculation we have assumed that the line is optically thin for reasons that will be explained in Sect. 5.2. The dynamical time scale, obtained from the ratio between the length of the lobes, and the maximum speed in the line wings, is $\sim 1.6 \times 10^{3} \mathrm{yr}$, which is only a lower limit of the MYSO age. We use 0.' 6 (or $0.018 \mathrm{pc}$ ) as the length of the lobes, estimated from the separation between the peaks of the blueand red-shifted maps of the $\mathrm{NH}_{3}$ emission (see Fig. 8). For the maximum speed in the line wings, we take $6.3 \mathrm{~km} \mathrm{~s}^{-1}$, which was evaluated taking the difference in velocity between the most blue- and red-shifted channels where ammonia emission is detected. We caution that the derived dynamical time scale is not corrected for the (unknown) inclination, $i_{0}$, of the outflow with respect to the line of sight. Such a correction, corresponding to the factor $\operatorname{coth}\left(i_{\mathrm{O}}\right)$, becomes important if the outflow is close to the plane of the sky or the line of sight. The dynamical time scale measured for the NE-SW outflow by López-Sepulcre et al. (2009) using CO single-dish observations is $\approx 10^{4} \mathrm{yr}$, significantly larger than the value determined from the $\mathrm{NH}_{3}$ data and likely close to the MYSO age. For our estimates we used a gas temperature of $140 \mathrm{~K}$, equal to the maximum brightness temperature measured in the $\mathrm{NH}_{3}$ line. This is consistent with the rotational temperature $\left(130_{-23}^{+36} \mathrm{~K}\right)$ computed by F2008 from the $\mathrm{CH}_{3} \mathrm{CN}(5-4)$ lines. Note that reducing the temperature to, for example, $50 \mathrm{~K}$ would decrease the outflow parameters only by a factor $\sim 2$.

We obtain $\dot{M}_{\text {out }} \simeq 3 \times 10^{-7} / X M_{\odot} \mathrm{yr}^{-1}, \dot{P}_{\text {out }} \simeq 2 \times$ $10^{-11} / X M_{\odot} \mathrm{km} \mathrm{s}^{-1} \mathrm{yr}^{-1}, \dot{E}_{\text {out }} \simeq 7 \times 10^{-7} / X L_{\odot}$, where $X$ is the relative abundance of $\mathrm{NH}_{3}$ with respect to $\mathrm{H}_{2}$. For a fiducial $X=4 \times 10^{-8}$ (see e.g., Van Dishoek et al. 1993; Sánchez-Monge et al. $2013 \mathrm{c}$, and references therein), the value of $\dot{P}(5 \times$ $10^{-4} M_{\odot} \mathrm{km} \mathrm{s}^{-1} \mathrm{yr}^{-1}$ ) is only a few times less than that estimated for the NE-SW outflow by López-Sepulcre et al. (2009) from the ${ }^{13} \mathrm{CO}(2-1)$ line $\left(0.82-2 \times 10^{-3} M_{\odot} \mathrm{km} \mathrm{s}^{-1} \mathrm{yr}^{-1}\right)$. Such a discrepancy could be explained by the uncertainty on the ammonia abundance and we thus conclude that ammonia might be tracing the root of the larger scale CO outflow oriented NE-SW.

\subsubsection{The two-clump scenario}

We also consider another explanation for the two ammonia components, namely that they are tracing two distinct, nearby clumps with slightly different velocities. To investigate this possibility, we have analyzed the $\mathrm{N}_{2} \mathrm{H}^{+}(1-0)$ data of F2008 again. $\mathrm{N}_{2} \mathrm{H}^{+}$is found in close association with $\mathrm{NH}_{3}$ and the OVRO maps obtained by F2008 are sensitive to more extended emission than the higher-resolution ammonia maps obtained by us. We have fit the $\mathrm{N}_{2} \mathrm{H}^{+}$spectra taking into account the hyperfine splitting of the transition and created maps of the average emission over the

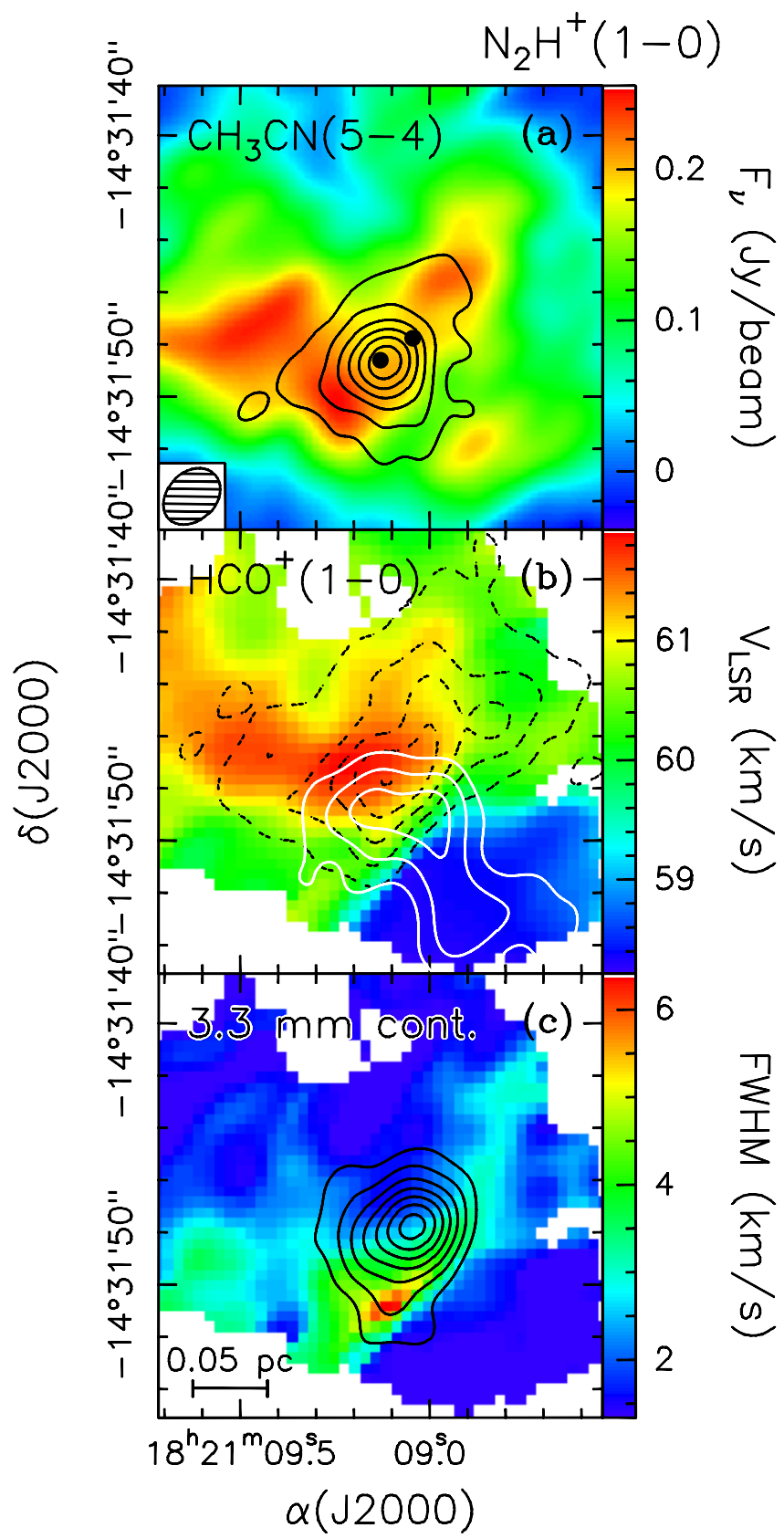

Fig. 9. Obtained from the data of F2008: a) Map of the $\mathrm{CH}_{3} \mathrm{CN}(5-4)$ line emission (contours) tracing the HMC, overlaid on a map of the $\mathrm{N}_{2} \mathrm{H}^{+}(1-0)$ emission (from F2008) averaged over the main line (color scale). The black dots indicate the positions of the centimeter sources "a" (alias mm-core) and "b" (alias HMC), detected by Zapata et al. (2006). b) Maps of the blue- (solid white contours) and red-shifted (dashed black) emission of the $\mathrm{HCO}^{+}(1-0)$ line overlaid on a map of the $\mathrm{N}_{2} \mathrm{H}^{+}(1-0)$ line velocity. The $\mathrm{HCO}^{+}(1-0)$ maps are derived using the most extreme velocity channels $\left(1.68 \mathrm{~km} \mathrm{~s}^{-1}\right.$ wide) at which emission was detected by F2008, centered at $55.8 \mathrm{~km} \mathrm{~s}^{-1}$ and $62.5 \mathrm{~km} \mathrm{~s}^{-1}$ for the blue- and red-shifted line, respectively. c) Map of the $3.3 \mathrm{~mm}$ continuum emission (contours) tracing the mm-core, overlaid on a map of the $\mathrm{N}_{2} \mathrm{H}^{+}(1-0)$ line FWHM.

main line, peak velocity, and line FWHM. These are shown, respectively, in Figs. 9a,b,c. Overlaid on these, we also show contour maps of the $\mathrm{CH}_{3} \mathrm{CN}(5-4)$ line emission, $\mathrm{HCO}^{+}(1-0)$ blueand red-shifted emission, and $3.3 \mathrm{~mm}$ continuum emission.

The most striking feature is the sharp change in velocity seen in Fig. 9b along a NE-SW line, which looks consistent with the 
bipolar structure seen in $\mathrm{HCO}^{+}$. This result weakens the outflow interpretation significantly, because $\mathrm{N}_{2} \mathrm{H}^{+}$is a well known tracer of dense gas, but is only exceptionally detected in outflows (see the case of L1157; Tobin et al. 2011). Moreover, for a bipolar outflow one would not expect such a sharp change in velocity because the line peak should shift from red to blue velocities gradually, going from one lobe to the other. We thus believe that the existence of two clumps is at least as likely as the outflow scenario. Indeed, looking at Fig. 9a, one sees that while most of the emission outlines a big, complex clump to the NE (where the compact $\mathrm{CH}_{3} \mathrm{CN}$ core is located), a minor peak of emission is also seen to the SW, possibly tracing another less massive clump.

Let us hence assume for the moment that we are dealing with two nearby clumps moving with different velocities along the line of sight. The question is whether these two clumps are physically interacting or only overlapping in projection on the plane of the sky. Comparison between Figs. 9c,b reveals that the line FWHM attains a maximum just along the line where the sudden velocity change is seen. The fact that such a maximum coincides with a tail of millimeter continuum emission (contours in Fig. 9c) seems to confirm that the FWHM increase is not due to two velocity components overlapping along the line of sight, but to a real enhancement of the column density. Although hazardous at this stage, one might even speculate that the formation of the mm-core has been triggered by the collision of the two clumps, as it has been observed in other regions (Duarte-Cabral et al. 2011; Henshaw et al. 2013).

Clearly, further observational evidence is needed before drawing any conclusion on the nature of the velocity field in this region. However, if the two-clump hypothesis were correct, this could imply that the NE-SW bipolar outflow claimed by B2006 and López-Sepulcre et al. (2009) does not exist.

The scenarios proposed here and in the Sect. 5.1.1 will be further discussed in Sect. 6.

Before that, however, we wish to analyze the velocity field of the ammonia gas in some better detail, searching for a connection with the $\mathrm{CH}_{3} \mathrm{OH}$ maser disk proposed by $\mathrm{S} 2010$.

\subsection{Ammonia from the $\mathrm{CH}_{3} \mathrm{OH}$ maser disk}

Our data make it possible to find out whether the maser disk proposed by $\mathrm{S} 2010$ is detected also in the ammonia emission. The most direct approach consists in studying the gas velocity along the disk plane by means of a position-velocity plot of the $\mathrm{NH}_{3}$ emission. Since the $\mathrm{NH}_{3}(1,1)$ main line is slightly blended with the inner satellites (see Fig. 7) and the $(1,1)$ transition traces more extended (and colder) regions than the $(2,2)$ line, we have chosen the latter for our purposes. The plane of the disk is assumed to lie along the direction joining the centers of the redand blue-shifted maser clusters (see e.g., Fig. 8), i.e., along a PA of $140^{\circ}$.

The position-velocity plot of the $\mathrm{NH}_{3}(2,2)$ main line is shown in Fig. 10, where we also report the points representing the $\mathrm{CH}_{3} \mathrm{OH}$ maser spots. One sees that ammonia emission is indeed detected at the same positions and velocities as the two groups of masers, consistent with emission from a rotating disk/ring. However, the emitting region is not limited to the locations of the masers, but extends up to an offset of $\sim 0$ '. $^{\prime}$ to the NW. Here the $\mathrm{NH}_{3}$ emission appears to attain its maximum, both at blue- and red-shifted velocities. Such an offset between the methanol masers center and the location of the two ammonia velocity components was already pointed out in Sect. 5.1 (see also Fig. 8). In fact, the position-velocity plot along the direction perpendicular to the disk plane and passing through the

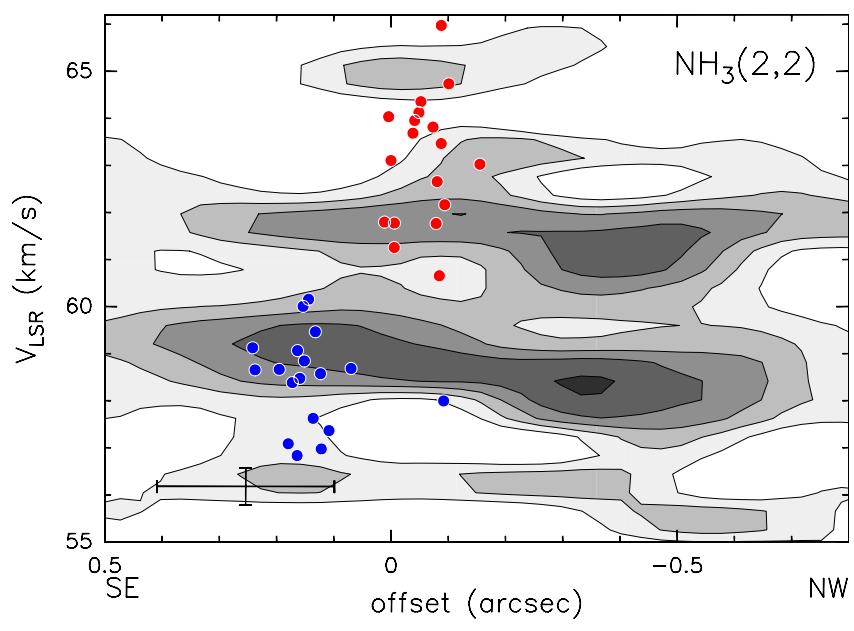

Fig. 10. Position-velocity plot of the $\mathrm{NH}_{3}(2,2)$ line emission along a direction crossing the centers of the red- and blue-shifted $\mathrm{CH}_{3} \mathrm{OH}$ maser clusters $\left(\mathrm{PA}=140^{\circ}\right)$. Contour levels range from 2 to $6 \mathrm{mJy} / \mathrm{beam}$ in steps of $1 \mathrm{mJy} / \mathrm{beam}$. The red and blue circles represent the methanol maser spots. The cross in the bottom left indicates the angular and spectral resolutions.

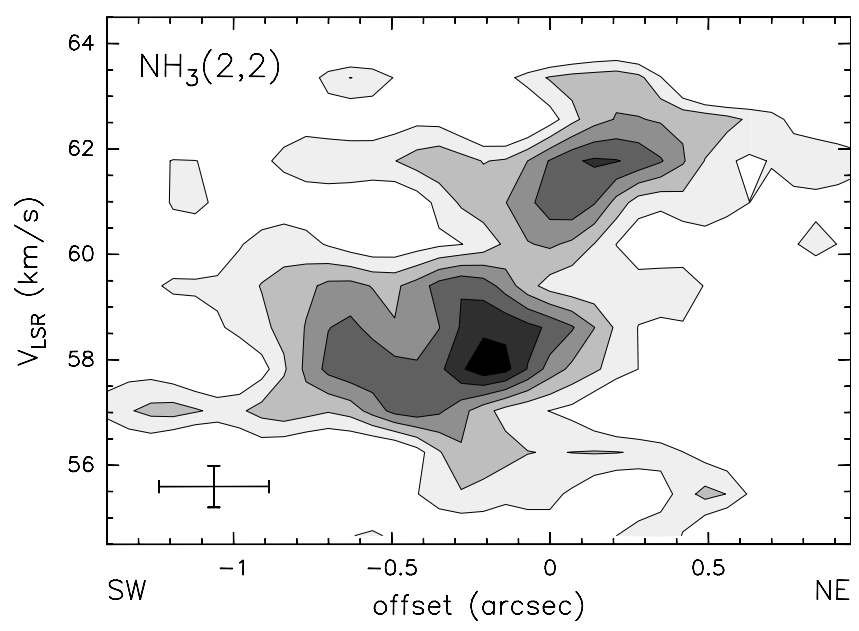

Fig. 11. Position-velocity plot of the $\mathrm{NH}_{3}(2,2)$ line emission along a direction perpendicular to that of Fig. 10 (i.e., with PA $=50^{\circ}$ ) and passing at an offset of 0.4 to the NW of the $\mathrm{CH}_{3} \mathrm{OH}$ masers. Contour levels range from 2 to $7 \mathrm{mJy} /$ beam in steps of $1 \mathrm{mJy} /$ beam. The cross in the bottom left indicates the angular and spectral resolutions.

ammonia peak (i.e., at an offset of $0 . ' 4$ to the NW of the maser center) clearly outlines the two velocity components, as shown in Fig. 11.

We conclude that the methanol maser disk is detected also in the ammonia emission, although quite faint.

\section{A model for the $\mathrm{G} 16.59-0.05$ region}

Before proposing an interpretation for the G16.59-0.05 starforming region, it is worth summarizing the observational findings obtained in this and previous studies:

- A compact $(\sim 0.04 \mathrm{pc})$ dusty core is detected at millimeter wavelengths. Hot-core tracers such as $\mathrm{CH}_{3} \mathrm{CN}$ and $\mathrm{NH}_{3}$ peak at a position offset by $\sim 1^{\prime \prime} .3(\sim 0.022 \mathrm{pc})$ to the SE from the core center.

- Three mid-IR sources are seen within $\sim 15^{\prime \prime}$ from the HMC, but only that associated with the HMC appears to dominate 
the bolometric luminosity of the whole region $\left(\sim 10^{4} L_{\odot}\right.$, corresponding to a $\sim 13 M_{\odot}$ ZAMS star).

- The detection of two outflows extending over $\sim 20^{\prime \prime}(0.35 \mathrm{pc})$ has been claimed by various authors, one directed NE-SW (B2006; López-Sepulcre et al. 2009) and the other SE-NW (B2006; F2008).

- Our ammonia observations have detected two velocity components arising from a compact region $(\sim 0)^{\prime} 6$ or $\left.0.01 \mathrm{pc}\right)$. We propose that these might be tracing either the root of the bipolar outflow directed NE-SW, or the region of overlap between two clumps moving with different velocities along the line of sight.

- A radio jet directed E-W is imaged from 6 to $1.3 \mathrm{~cm}$. While the $6 \mathrm{~cm}$ continuum is dominated by non-thermal (possibly synchrotron) emission, at $1.3 \mathrm{~cm}$ compact free-free emission appears to arise very close to the center of the group of $\mathrm{CH}_{3} \mathrm{OH}$ maser spots detected by S2010. A $10 M_{\odot}$ ZAMS star is sufficient to explain the observed flux in terms of ionization by the stellar Lyman continuum - although ionization by shocks along the radio jet cannot be excluded.

- The $\mathrm{H}_{2} \mathrm{O}$ maser proper motions measured by S2010 seem to trace expansion to the west, along the western border of the radio jet. The $\mathrm{CH}_{3} \mathrm{OH}$ maser spots cluster in two groups that appear to rotate about the free-free continuum peak, with a rotation axis oriented NE-SW and an equilibrium mass of $\sim 12 M_{\odot}$ (from S2010, after correcting for the new distance estimate). A similar velocity field is seen also in the ammonia lines.

We have proposed two scenarios to interpret the $\mathrm{NH}_{3}$ and $\mathrm{N}_{2} \mathrm{H}^{+}$ observations and in the following we will refer to them as "case A", for that depicted in Sect. 5.1.1 (ammonia emission associated with the NE-SW bipolar outflow) and "case B", for that illustrated in Sect. 5.1.2 (ammonia emission from two overlapping clumps with different velocities). Figure 12 shows a sketch that summarizes both scenarios.

Neither case A nor case B can be explained with only one dominant MYSO in the G16.59-0.05 region. In fact, case B implies the existence of a radio jet associated with a $\mathrm{CH}_{3} \mathrm{OH}$ maser disk (see Sect. 3), plus the SE-NW outflow detected by B2006 and F2008. On the one hand, methanol masers have never been found in association with low-mass YSOs; on the other, the momentum rate of the SE-NW outflow is typical of MYSOs. We thus conclude that both the radio jet and the outflow must be powered by MYSOs. In this scenario the disk-jet system is associated with the HMC, while the outflow might be originating from a young massive protostar deeply embedded in the $\mathrm{mm}$-core.

The situation of case A is more complex. Here we have the disk-jet system, the SE-NW outflow, and the NE-SW outflow that we detect in ammonia on a small scale. Like in case B, the (so far undetected) MYSO powering the SE-NW outflow might be lying inside the mm-core. As for the radio jet and NE-SW outflow, we propose that both could be associated with the same MYSO. The problem with this hypothesis are the different directions of the E-W jet and NE-SW outflow. This discrepancy can be explained if the jet/outflow is undergoing precession, because the direction of the jet/outflow axis would change from the small to the large scale, as in the case of the massive protostar IRAS 20126+4104 (Shepherd et al. 2000; Cesaroni et al. 2005). Alternatively, the apparent direction of the radio jet might be affected by the density distribution around the YSO powering the jet, as the density appears to be much higher to the west where the core is located. As already noted in Sect. 5.1, such

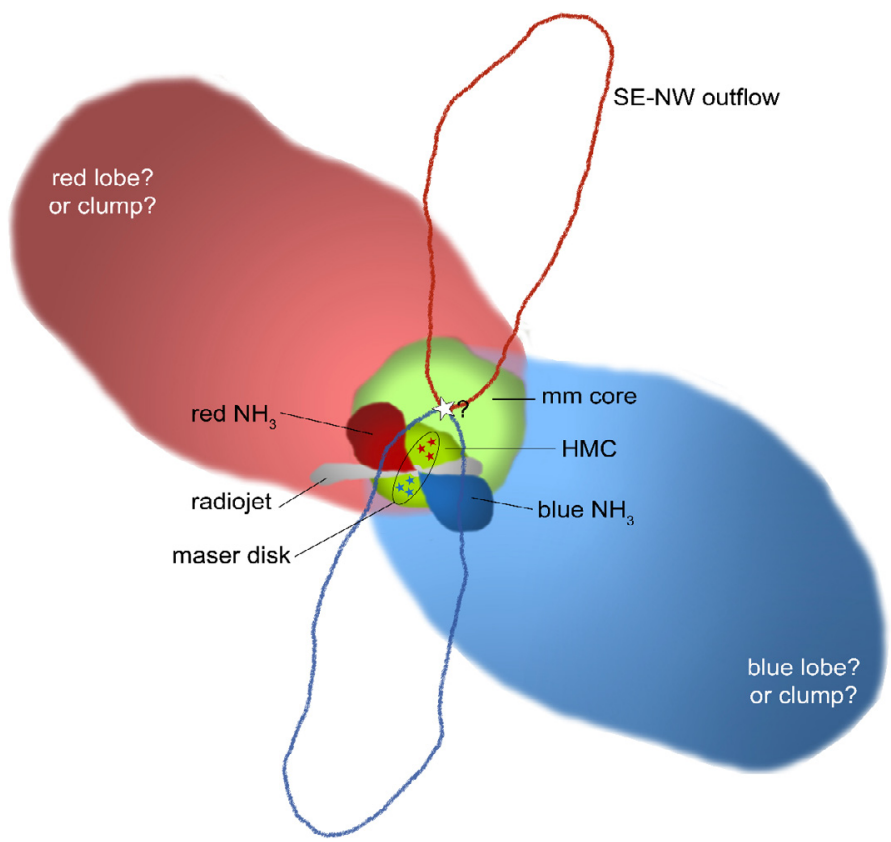

Fig. 12. Sketch of the scenarios proposed to explain all the features observed in the G16.59-0.05 region. Note that the relative sizes of the different structures (disk, jet, outflow, etc.) have been arbitrarily chosen to make the figure more readable.

an asymmetric distribution could also justify the small offset observed between the $\mathrm{CH}_{3} \mathrm{OH}$ masers and the geometrical center of the $\mathrm{NH}_{3}$ flow (see Fig. 8).

Is the case-A scenario compatible with the physical parameters estimated by us? We note that the values of the stellar mass (10-13 $\left.M_{\odot}\right)$ derived from the bolometric luminosity, $\mathrm{CH}_{3} \mathrm{OH}$ maser rotation velocity, and free-free $1.3 \mathrm{~cm}$ continuum, are all in good agreement with each other. Using models of outflow evolution recently presented by Duarte-Cabral et al. (2013), a star of final mass of $10-15 M_{\odot}$, evolved to the stage of a HMC, should emit outflows with typical momentum rates of a few $10^{-4} M_{\odot} \mathrm{km} \mathrm{s}^{-1} \mathrm{yr}^{-1}$. Considering the uncertainty on the inclination angle of the outflow, such a value agrees well with the momentum rate, $5 \times 10^{-4} M_{\odot} \mathrm{km} \mathrm{s}^{-1} \mathrm{yr}^{-1}$, we have derived in Sect. 5.1.1 for the $\mathrm{NH}_{3}$ flow.

Our conclusion is that the observational evidence collected so far is insufficient to decide between case A and B, although we tend to favor the latter because case $\mathrm{A}$ implies the existence of a NE-SW outflow traced also by the $\mathrm{N}_{2} \mathrm{H}^{+}(1-0)$ line: to our knowledge, this would be the second case ever of $\mathrm{N}_{2} \mathrm{H}^{+}$tracing an outflow - and the very first case for outflows from MYSOs.

\section{Summary and conclusions}

We performed ammonia line and radio and IR continuum observations of the G16.59-0.05 star-forming region with subarcsecond resolution, to shed light on the structure and velocity field of the mm-core and $\mathrm{HMC}$ nearby the $\mathrm{CH}_{3} \mathrm{OH}$ maser disk imaged by S2010, and establish the origin of the two bipolar outflows observed in the region. We discover a radio jet oriented E-W and centered on the $\mathrm{CH}_{3} \mathrm{OH}$ masers, whose western lobe appears to expand to the west, impinging against the $\mathrm{HMC}$, as suggested by the $\mathrm{H}_{2} \mathrm{O}$ maser proper motions measured by $\mathrm{S} 2010$. The radio continuum from the jet has negative spectral index between 6 and $2 \mathrm{~cm}$, indicating non-thermal continuum 
(synchrotron) emission, with the sole exception of a compact free-free continuum source close to the $\mathrm{CH}_{3} \mathrm{OH}$ masers.

Our $24.5 \mu \mathrm{m}$ image complemented by Herschel continuum data from the Hi-GAL survey indicate that the bolometric luminosity of the region is dominated by the emission from the neighborhoods of the HMC and mm-core. The stellar mass implied by the observed luminosity $\left(\sim 13 M_{\odot}\right)$ is in good agreement with both the equilibrium mass of the $\mathrm{CH}_{3} \mathrm{OH}$ maser disk $\left(\sim 12 M_{\odot}\right)$, and the stellar mass $\left(\gtrsim 10 M_{\odot}\right)$ needed to explain the free-free continuum emission - assuming the latter is due to ionization by stellar photons.

We find that the ammonia emission presents a bipolar structure consistent (on a smaller scale) in direction and velocity with that of the NE-SW bipolar outflow reported by B2006 and López-Sepulcre et al. (2009). After analyzing the $\mathrm{N}_{2} \mathrm{H}^{+}(1-0)$ observations of F2008 again, we conclude that two scenarios are possible. Both imply the existence of two MYSOs, but differ in the interpretation of the NE-SW bipolar structure. In one case this is a bipolar outflow (in agreement with B2006 and López-Sepulcre et al. 2009), which represents the prosecution on the large scale of the small-scale radio jet. In the other case, the bipolarity is interpreted as two overlapping clumps moving with different velocities along the line of sight. We slightly favor the second hypothesis, because the first would imply that the NE-SW outflow is detected also in the $\mathrm{N}_{2} \mathrm{H}^{+}(1-0)$ line emission, which appears unlikely. Discriminating between the two scenarios requires observations with higher sensitivity than our VLA ammonia images and we believe that the Atacama Large Millimeter Array will be the right instrument to establish the nature of the NE-SW bipolar structure and investigate the circumstellar disk associated with the methanol masers.

Acknowledgements. We thank the anonymous referee for his constructive critcisms and the suggestion to analize the $\mathrm{N}_{2} \mathrm{H}^{+}$data by F2008 again, which helped us to shed new light on this complex region. R.S.F. acknowledges T. Usuda, T. Inagaki, S. S. Hayashi, and H. Shinnaga for their help with the Subaru observations and data reduction. We are greatful to Beatriz Sánchez Monge and Rafael Delgado Romero for preparing the sketch shown in Fig. 12. A. Sanna acknowledges the financial support by the European Research Council for the ERC Advanced Grant GLOSTAR under contract no. 247078.

\section{References}

Anglada, G. 1996, in Radio Emission from the Stars and the Sun, eds. A. R. Taylor, \& J. M. Paredes, ASP Conf. Ser., 93, 3

Benjamin, R. A., Churchwell, E., Babler, B. L., et al. 2003, PASP, 115, 953

Beuther, H., Schilke, P., Menten, K. M., et al. 2002a, ApJ, 566, 945

Beuther, H., Schilke, P., Sridharan, T. K., et al. 2002b, A\&A, 383, 892

Beuther, H., Zhang, Q., Sridharan, T. K., Lee, C.-F., \& Zapata, L. A. 2006, A\&A, 454, 221 (B2006)
Carey, S. J., Noriega-Crespo, A., Mizuno, D. R., et al. 2009, PASP, 121, 76

Carrasco-González, C., Rodríguez, L. F., Anglada, G., et al. 2010, Science, 330, 1209

Cesaroni, R., Neri, R., Olmi, L., et al. 2005, A\&A, 434, 1039

Cesaroni, R., Galli, D., Lodato, G., Walmsley, C. M., \& Zhang, Q. 2007. Protostars and Planets V, 197

Cohen, M., Walker, R. G., Carter, B., et al. 1999, AJ, 117, 1864

Contreras, Y., Schuller, F., Urquhart, J. S., et al. 2013, A\&A, 549, A45

Cunningham, A. J., Klein, R. I., Krumholz, M. R., \& McKee, C. F. 2011, ApJ, 740,107

Davies, B., Hoare, M. G., Lumsden, S. L., et al. 2011, MNRAS, 416, 972

De Buizer, J. M., Radomski, J. T., Telesco, C. M., \& Piña, R. K. 2005, ApJS, 156,179

Drosback, M. M., Aguirre, J., Bally, J., et al. 2008, in BAAS, 40, Am. Astron. Soc. Meet. Abstr. \#212, 271

Duarte-Cabral, A., Dobbs, C. L., Peretto, N., \& Fuller, G. A. 2011, A\&A, 528, A50

Duarte-Cabral, A., Bontemps, S., Motte, F., et al. 2013, A\&A, 558, A125

Egan, M. P., Price, S. D., \& Kraemer, K. E. 2003, in BAAS, 35, Am. Astron. Soc. Meet. Abstr., 1301

Furuya, R. S., Cesaroni, R., Takahashi, S., et al. 2008, ApJ, 673, 363 (F2008)

Ghavamian, P., \& Hartigan, P. 1998, ApJ, 501, 687

Henshaw, J. D., Caselli, P., Fontani, F., et al. 2013, MNRAS, 428, 3425

Hill, T., Burton, M. G., Minier, V., et al. 2005, MNRAS, 363, 405

Kataza, H., Okamoto, Y., Takubo, S., et al. 2000, in SPIE Conf. Ser., 4008, eds. M. Iye, \& A. F. Moorwood, 1144

Keto, E. 2002, ApJ, 580, 980

Keto, E. 2003, ApJ, 599, 1196

López-Sepulcre, A., Codella, C., Cesaroni, R., Marcelino, N., \& Walmsley, C. M. 2009, A\&A, 499, 811

Molinari, S., Swinyard, B., Bally, J., et al. 2010a, A\&A, 518, L100

Molinari, S., Swinyard, B., Bally, J., et al. 2010b, PASP, 122, 314

Moscadelli, L., Sanna, A., \& Goddi, C. 2011, A\&A, 536, A38

Neugebauer, G., Habing, H. J., van Duinen, R., et al. 1984, ApJ, 278, L1

Panagia, N., \& Felli, M. 1975, A\&A, 39, 1

Peters, T., Mac Low, M.-M., Banerjee, R., Klessen, R. S., \& Dullemond, C. P. 2010, ApJ, 719, 831

Reid, M. J., Argon, A. L., Masson, C. R., Menten, K. M., \& Moran, J. M. 1995, ApJ, 443, 238

Reynolds, S. P. 1986, ApJ, 304, 713

Rodríguez, L. F., González, R. F., Montes, G., et al. 2012, ApJ, 755, 152

Sánchez-Monge, Á., Cesaroni, R., Beltrán, M. T., et al. 2013a, A\&A, 552, L10

Sánchez-Monge, Á., Kurtz, S., Palau, A., et al. 2013b, ApJ, 766, 114

Sanna, A., Moscadelli, L., Cesaroni, R., et al. 2010, A\&A, 517, A71 (S2010)

Schuller, F., Menten, K. M., Contreras, Y., et al. 2009, A\&A, 504, 415

Shepherd, D. S., Yu, K. C., Bally, J., \& Testi, L. 2000, ApJ, 535, 833

Tobin, J. J., Hartmann, L., Chiang, H.-F., et al. 2011, ApJ, 740, 45

Williams, S. J., Fuller, G. A., \& Sridharan, T. K. 2005, A\&A, 434, 257

Zapata, L. A., Rodríguez, L. F., Ho, P. T. P., Beuther, H., \& Zhang, Q. 2006, AJ, 131, 939

Zhang, Q., Hunter, T. R., Sridharan, T. K., \& Cesaroni, R. 1999, ApJ, 527, L117 